\title{
Performance of DCSK System with Blanking Circuit for Power-Line Communications
}

\author{
Georges Kaddoum, Member, IEEE \\ LACIME Laboratory* \\ École de technologie supérieure \\ Montréal, Canada \\ georges.kaddoum@etsmtl.ca
}

\author{
Navid Tadayon, Member, IEEE \\ LACIME Laboratory \\ École de technologie supérieure \\ Montréal, Canada \\ tadayon@emt.inrs.ca
}

\author{
Ebrahim Soujeri, Member, IEEE \\ LACIME Laboratory \\ École de technologie supérieure \\ Montréal, Canada \\ Ebrahim.Soujeri@lacime.etsmtl.ca
}

\begin{abstract}
Providing from the robustness of differential chaos shift keying (DCSK) against linear and non-linear channel distortions, in this paper, we propose using this non-coherent modulation with blanking device as a potential candidate for implementation in power-line communications. The performance of this system is analyzed in the general case where background, impulsive and phase noises exist. We derive the probability of blanking error as well as the bit error rate with and without blanking. Moreover, the proposed system is compared to differential phase shift keying (DPSK) modulation to highlight the robustness of DCSK scheme against phase noise imperfection.
\end{abstract}

\section{INTRODUCTION}

Power line communication (PLC) has been long used to transmit data over available power lines. The passion and enthusiasm into this field has lately been restored as PLC emerged as a capable rival in the broadband communication market for indoor in-building networking and also for the lastmile access [1]. Some features like plug-and-play connection, always-on access, higher data rate support as well as the application to remote and rural areas are only few out of many advantages that PLC can offer [2]. Standardization groups such as IEEE P.1901 and ETSI PLT are currently working on PLC to come up with effective modulation schemes for the imminent commercialization of PLC [3]. As a result of all these efforts, quite a good number of modulation schemes have been recommended to transmit digital information over PLC channels. Unlike the case of low data rate applications where phase and frequency shift keying are sufficient, for high data rate applications, orthogonal frequency division multiplexing (OFDM) as well as code division multiple access (CDMA) have been standing out as potential solutions for adoption into future PLC architectures [4]. The data transmission on electrical lines is not as easy as it seems. Indeed, the existence of unpredictable factors, whose power spectral density (PSD) span through wide bands of spectrum, can easily render communication over power lines impossible. Some of these factors that are mostly of noise/interference nature stemming from different sources and exhibiting different behaviours are stationary, background noise [5] and time-variant, impulsive noise [5]. The latter is the most threatening degradation with short \footnotetext{
2013.

* This work has been supported by the NSERC discovery grant $435243-$
}

(C) 2016 IEEE. Personal use of this material is permitted. Permission from IEEE must be obtained for all other uses, in any current or future media, including reprinting/republishing this material for advertising or promotional purposes, creating new collective works, for resale or redistribution to servers or lists, or reuse of any copyrighted component of this work in other works. duration and random occurrence nature associated with a high power spectral density. The blanking method that is introduced in this study can nullify the effects of impulsive noise [6] to a large extent. Various chaos-based communication systems with both coherent and non-coherent detection have been proposed in the last decade [7]-[10]. This is due to the fact that, chaotic signals have instinctive wideband characteristics that make them a very well fit for various spread spectrum modulation schemes. In fact, similar to other spread spectrum modulation techniques, chaos-based modulations have advantages such as reduction of fading effects and jamming resistance [8], [9]. Differential chaos shift keying (DCSK) is the mostly promising scheme among chaos-based communication schemes [7]. The fundamental reason for such widespread adoption is that DCSK require neither chaotic synchronization nor knowledge of the channel state information (CSI) at the receiver [7], [11]. By the same token, the DCSK is more immune to multipath fading and to nonlinear distortions, when compared to the DPSK scheme, and is much more suitable for ultra wide band (UWB) applications [7], [8], [11].

In this paper, we investigate the application of DCSK modulation using blanking device at the receiver for power line communication. To the best of our knowledge, there is no previous study analysing such class of modulation in PLC systems. It is important to note that the DCSK system is chosen in this paper as a benchmark for transmit reference noncoherent chaos-based communication systems. In detailing our contrition in this paper, in Section II the DCSK modulation scheme and blanking device is proposed. In section III, the analytical expression of probability of blanking error is derived as well as the bit error rate probability expressions of the system in the presence of high impulsive noise power, phase noise and with and without the blanking device. Moreover, we discuss the threshold level on the performance of the system and we validate our analytical expressions by computer simulations. In section IV, the performance of DPSK and DCSK is compared through simulation in order to show the resistance of the latter to phase noise interference.

\section{DCSK SYSTEM WITH BLANKING DEVICE}

Fig. 1 is a diagrammatic representation of the general structure of DCSK communication system where chaotic samples 
spread a given information bit $b_{i}$. At the output of the DCSK modulator, each bit $b_{i}= \pm 1$ is composed of two equal portions, each with length equal of $\beta$; the first is the reference portion which only consists of the chaotic sequence samples. The second is the data-carrying portion which consists of the multiplication of chaotic sequences sequences with the incoming bit stream $b_{i}$. If $b_{i}=+1$ is to be transmitted, the data-bearing sequence is exactly the reference sequence, and if $b_{i}=-1$ is to be sent, the data-carrying sequence is an inverted version of the reference sequence. The spreading factor in DCSK systems is defined as the number of chaotic samples used to spread each bit and is presented by $2 \beta$, where $\beta$ is an integer. Moreover, $T_{D C S K}=2 T_{b}=2 \beta T_{c}$ is the DCSK frame time interval for each bit and $T_{c}$ is the chip interval. During the $i^{\text {th }}$ bit time interval, the discrete form of the discrete baseband signal sequence at the output of the transmitter $s_{k, i}$ can be given by

$$
s_{k, i}= \begin{cases}x_{k, i}, & 0<k \leq \beta, \\ b_{i} x_{k-\beta, i}, & \beta<k \leq 2 \beta,\end{cases}
$$

where $x_{k, i}$ is the chaotic sequence used as the reference signal and $x_{k-\beta, i}$ is its delayed version. After travelling through the PL channel, the received signal $r_{k, i}$ is corrupted with impulsive and background noise [5]. Hence, the total noise can be modelled as

$$
n_{k, i}=w_{k, i}+I_{k, i},
$$

where $n_{k, i}$ is the total noise, $w_{k, i}$ is the complex additive white Gaussian noise (AWGN) and $I_{k, i}$ is the impulsive complex noise affecting $i^{\text {th }}$ bit at time $k$. It is important to note that the different signal components present in equation (2) are mutually independent. In this paper, we consider the twomixture Gaussian model [12] in our analysis.

$$
I_{k, i}=B_{k, i} g_{k, i},
$$

where $g_{k, i}$ is a zero mean white Gaussian noise and $B_{k, i}$ is the Bernoulli process with probability $P\left(B_{k, i}=1\right)=p$, where $p$ represents the probability of occurrence of the impulsive noise. Hence, the total probability density function (PDF) of the sum of the noise signals $w_{k, i}$ and $I_{k, i}$ in (2) can be expressed as $f\left(n_{k}\right)=(1-p) \mathcal{N}\left(0, \sigma_{w}^{2}\right)+p \mathcal{N}\left(0, \sigma_{w}^{2}+\sigma_{I}^{2}\right)$. Here, $\mathcal{N}($.$) is the PDF of the Gaussian distribution. Also, \sigma_{w}^{2}$ is the background AWGN noise variance and $\sigma_{I}^{2}$ is the impulsive noise variance. We denote by signal-to-noise ratio (SNR) the ratio of the power of the signal $s_{k}$, i.e. $\sigma_{s}^{2}$, to the power of the AWGN noise $w_{k}$ represented by, $\mathrm{SNR}=10 \log _{10} \sigma_{s}^{2} / \sigma_{w}^{2}$. On the other hand, the signal-to-impulsive noise ratio (SINR) is given as $\operatorname{SINR}=10 \log _{10} \sigma_{s}^{2} / \sigma_{I}^{2}$. Whether the impulsive noise is present or not, at the receiver, the received signal $r_{k, i}$ of $i^{\text {th }}$ transmitted bit at time $k$ may be expressed as

$$
r_{k, i}=\left\{\begin{array}{ll}
s_{k, i} e^{j \phi_{i}}+w_{k, i} & \mathcal{H}_{0} \\
s_{k, i} e^{j \phi_{i}}+w_{k, i}+I_{k, i} & \mathcal{H}_{1}
\end{array},\right.
$$

where $\mathcal{H}_{0}$ and $\mathcal{H}_{1}$ represent the hypotheses of the absence and the presence of impulsive noise having the following probabilities $P\left(\mathcal{H}_{0}\right)=(1-p)$ and $P\left(\mathcal{H}_{1}\right)=p$ respectively.
The exponent $\phi_{i} \in[-\pi, \pi]$ in (4) is a uniformly distributed random variable representing the phase noise. It is important to note that this phase variable is maintained constant over more than one DCSK bit duration $T_{D C S K}$. As shown in Fig. 1 , the received signal is first fed into the blanking device to compare its magnitude to a given blanking threshold $T$. The vector signal $\mathbf{y}_{\mathbf{i}}$ of $2 \beta$ samples exiting the blanking process can then be expressed as

$$
\mathbf{y}_{\mathbf{i}}= \begin{cases}\mathbf{r}_{i}, & E\left[\left\|\mathbf{r}_{i}\right\|\right] \leq T \\ 0, & E\left[\left\|\mathbf{r}_{i}\right\|\right]>T\end{cases}
$$

where $E\left[\left\|\mathbf{r}_{i}\right\|\right]$ is the average of the absolute values of $2 \beta$ samples corresponding to the $i$ th received DCSK bit. Since the blanking device is in fact a magnitude-based comparison filter, the terms $\operatorname{ang}(\cdot)$ and $\exp (j)$ are utilized to guard and retain the information content of the phase of the received signal, as elaborated in Fig. 1. It should be acknowledged that the blanking threshold must be optimized to assure the best outcome because a low value of $T$ will wash out a large portion of the useful signal. Likewise, choosing $T$ too large will let in most of the impulsive noise which noticeably degrades the BER performance. In order to demodulate the transmitted bits, the signal $y_{k}$ at the output of the blanking device is correlated with its delayed complex conjugate version $y_{k+\beta}^{*}$ and summed over the duration $\beta T_{c}$. The received bits are then estimated by computing the sign of the real part of the correlator output. The decision variable $D_{i}$ of the $i^{\text {th }}$ bit at the output of the correlator when $I_{k}$ is absent becomes

$$
D_{i}=\Re\left(\sum_{k=1}^{\beta}\left(x_{k, i} e^{j \phi_{i}}+w_{k, i}\right)\left(b_{i} x_{k, i} e^{j \phi_{i}}+w_{k+\beta, i}\right)^{*}\right),
$$

where $\Re$ represents the real operator. This decision variable may be further expanded as

$$
\begin{aligned}
D_{i} & =\sum_{k=1}^{\beta} b_{i} x_{k, i}^{2}+\Re\left(\sum_{k=1}^{\beta} x_{k, i}\left(w_{k+\beta, i}^{*} e^{j \phi_{i}}+b_{i} w_{k, i} e^{-j \phi_{i}}\right)\right) \\
& +\Re \sum_{k=1}^{\beta}\left(w_{k+\beta, i}^{*} w_{k, i}\right) .
\end{aligned}
$$

Due to its easiness and good performance, the second-order Chebyshev polynomial function (CPF) $x_{k+1}=1-2 x_{k}^{2}$ is used to generate chaotic sequences. The variance of the normalized chaotic map with zero mean is equal to one, i.e. $V[x]=\mathrm{E}\left[x^{2}\right]=1$, where $\mathrm{E}[\cdot]$ denotes the probabilistic expectation operator. As is it implied from (7), the useful signal $b_{i} x_{k, i}^{2}$ in the decision variable is independent from the phase noise. This independence is due to the correlation between the received signal and its delayed complex conjugate replica.

\section{Performance Analysis of DCSK system over PLC CHANNEL}

\section{A. Blanking: False-alarm and mis-detection probabilities}

In this section we derive the probability of blanking error $P_{b}$ event which occurs when the magnitude of the received signal $A_{r}=E\left[\left\|\mathbf{r}_{i}\right\|\right]$ exceeds the blanking threshold $T$ in the 


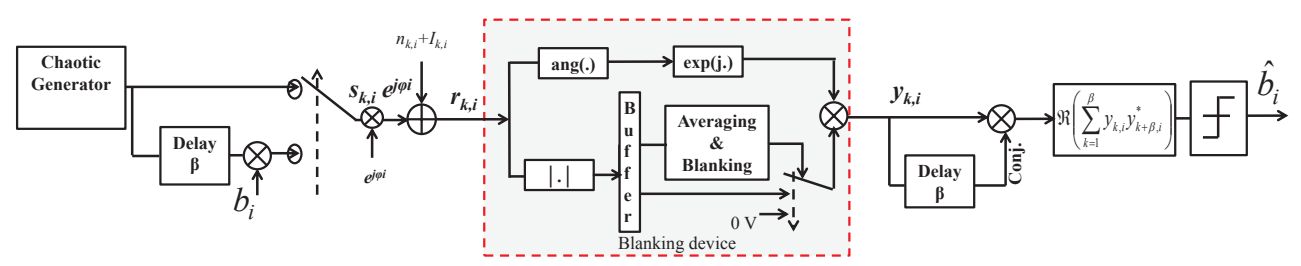

Fig. 1: Block diagram of the general structure of the DCSK communication system; (a) transmitter, receiver with blanking device.

absence of impulsive noise. This probability can be formulated as

$$
P_{b}=\operatorname{Pr}\left(A_{r}>T \mid \mathcal{H}_{0}\right) P\left(\mathcal{H}_{0}\right) .
$$

This expression leads to the probability of blanking error given by

$$
P_{b}=(1-p) \int_{T}^{\infty} f_{A_{r}}\left(r \mid \mathcal{H}_{0}\right) \mathrm{d} r
$$

where $f_{A_{r}}\left(r \mid H_{0}\right)$ is the PDF of the amplitude of the DCSK signal in the absence of impulsive noise. Additionally, the bit energy of the DCSK is not constant and varies from one bit to another [8] which makes the analytical expression of the PDF not available in closed-form. Instead, the solution of (9) can be derived numerically. In a similar manner, the probability of miss detection can be computed as

$$
P_{m}=p \int_{-\infty}^{T} f_{A_{r}}\left(r \mid \mathcal{H}_{1}\right) \mathrm{d} r
$$

where $f_{A_{r}}\left(r \mid H_{1}\right)$ is the PDF of the amplitude of the DCSK signal in presence of impulsive noise. In the same vein, the expression $P_{m}$ can only be derived numerically.

\section{$B$. Bit error rate probability}

From the above analysis, an error in the bit detection occurs in one of two cases; when the blanking device correctly predicts the absence of impulsive noise in the signal but the DCSK receiver makes an error in demodulating the bit; or when the system mis-detect the presence of the impulsive noise in the received signal and decodes the bits erroneously. Concluding these two cases, the total bit error rate with blanking device can be formulated as follows

$$
\mathrm{BER}_{\mathrm{Blank}}=\left(1-P_{m}\right) \mathrm{BER}_{\mathrm{BN}}+P_{m} \mathrm{BER}_{\mathrm{IBN}},
$$

where $B E R_{\text {Blank }}, \mathrm{BER}_{\mathrm{BN}}$ and $\mathrm{BER}_{\mathrm{IBN}}$ represent the total BER, the BER in the absence of impulsive noise and the BER in the presence of impulsive noise. For comparison, the total BER of the DCSK system without blanking is the sum the bit error rate in presence of background noise $\mathrm{BER}_{\mathrm{BN}}$ multiplied but the probability of absence of the impulsive noise and the BER in presence of background and impulsive noise $B R_{I B N}$ multiplied with the probability of occurrence $p$ of this latter.

$$
\mathrm{BER}_{\mathrm{PL}}=(1-p) \mathrm{BER}_{\mathrm{BN}}+p \mathrm{BER}_{\mathrm{IBN}}
$$

where $\mathrm{BER}_{\mathrm{PL}}$ is the total BER without the blanking device at the receiver side. When the impulsive noise is strong,
$\mathrm{BER}_{\mathrm{IBN}} \rightarrow 0.5$ in equations (11) and (12). To compute the BER expression in the absence of impulsive noise BER $_{\mathrm{BN}}$, the mean and the variance of the decision variable given in (7) should be derived. For the $i^{\text {th }}$ bit, the instantaneous mean of the decision variable is the mean of the useful signal given by

$$
E\left[D_{i}\right]=\frac{b_{i} E_{b}}{2}
$$

where $E_{b}=2 \sum_{k=1}^{\beta} E\left[x_{k, i}^{2}\right]$ is the total energy of the transmitted DCSK bit. Moreover, because the the interference components are independent, as are the the noise signal and is delayed version, the conditional variance of the two remaining interference components present in the decision variable for the $i^{\text {th }}$ bit can be expressed as

$$
V\left[D_{i}\right]=\frac{E_{b} N_{0} E\left[\cos \left(\phi_{i}\right)^{2}\right]}{2}+\beta \frac{N_{0}^{2}}{4} .
$$

Knowing that $E\left[\cos (\phi)^{2}\right]=0.5$ and using (13) and (14) above, the average BER of the DCSK scheme in the presence of background and phase noises is derived as

$$
\mathrm{BER}_{\mathrm{BN}}=\frac{1}{2} \int_{0}^{\infty} \operatorname{erfc}\left(\frac{2 N_{0}}{E_{b}}\left(1+\frac{\beta N_{0}}{E_{b}}\right)^{-0.5}\right) f\left(E_{b}\right) \mathrm{d}\left(E_{b}\right) .
$$

where $\operatorname{erfc}(x)$ is the complementary error function defined as $\operatorname{erfc}(x) \equiv 2 / \sqrt{\pi} \int_{x}^{\infty} e^{-u^{2}} d u$. and $f\left(E_{b}\right)$ is the PDF of the bit energy after spreading by chaotic signal.

\section{Discussions and Simulation Results}

In order to validate the performance of the DCSK scheme over power line channels, the analytical expressions for the probability of blanking error and BER are verified against simulation results. Fig. 2 shows the probability of blanking error of DCSK scheme for different SNR values versus the threshold $T$ in $\mathrm{dB}$. A careful examination of the performance curves shown in Fig. 2 reveals the extent to which simulation results perfectly validate the analytical expression provided in (9). For better performance, an adaptive threshold is required for such systems. In fact, even though lower blanking thresholds can reduce the probability of blanking error, but it increases re-transmissions of the bits nulled at the receiver side at the same time. The total bit error rate with blanking device is another important metric to be examined when analysing the performance. The obtained BER performance under power line communication channel for a spreading factor of $\beta=50$, $T=4.5$ and signal-to-impulsive noise ratio $\operatorname{SINR}=-2$ 


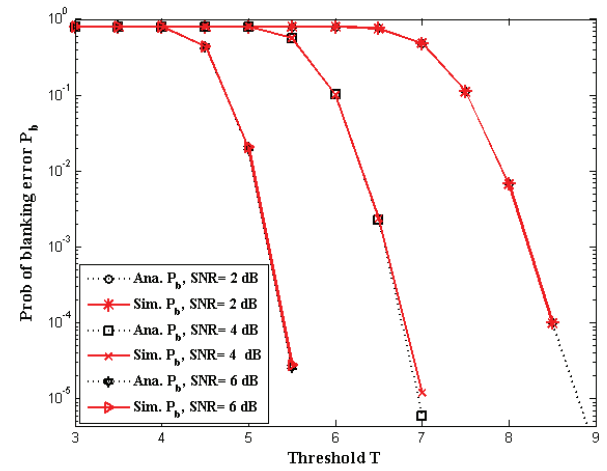

Fig. 2: Simulation, analytical probability of blanking error $\mathrm{Pb}$ with a spreading factor of $\beta=50$ and $p=0.2$.

$\mathrm{dB}$ with and without a blanking device and in presence of phase noise is shown in Fig. 3. The phase noise is assumed constant during 4 time symbols for both systems. Likewise, simulation results warrant the accuracy of the BER expression given in equations (11) and (12). As observed in Fig. 3, the

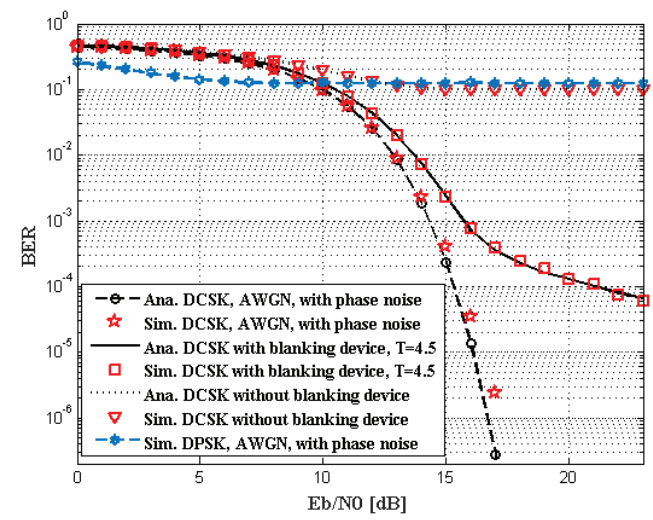

Fig. 3: Simulation, computed and BER for PLC channel with and without blanking device for a spreading factor of $\beta=50$ for $T=4.5$, SINR $=-2 \mathrm{~dB}$, and $p=0.2$.

proposed DCSK system with blanking device outperforms the conventional DCSK. In fact, this device eliminates the powerful impulsive noise by nulling the received signal. Naturally, when the impulsive noise affects many bits, the BER increases and the performance deteriorates. In addition, we observe a flooring in the total BER at high SNR. This comes from the miss-detected impulsive noise by the blanking circuit (lower flooring) or from the added impulsive noise when the system does not employ such a blanking circuit at all (upper flooring). Therefore, to preserve the quality of service of the transmission, it is better to re-transmit the affected bits/frames rather than decoding them with high probability of errors. This operation justifies the use of blanking device at the receiver side. Finally, we compared the BER performance of DCSK given in equation (15) and that of DPSK in the presence of phase noise and without the effect of the impulsive noise. As it is seen, the simulation again conforms to the analytical results.
Moreover, DCSK system exhibits a higher resistance to phase noise than DCSK. All these proves that our proposed scheme is a more suitable candidate for deployment in PLC scenarios.

\section{CONCLUSIONS}

In this study, a blanking device with DCSK receiver was proposed for power-line communication. Our proposed scheme enhances the overall system performance in presence of impulsive and phase noise. Instead of decoding the corrupted bits with impulsive noise with a high probability of error, the blanking device nulls the received signal when this latter appears. Moreover, the performance of the DCSK is analysed. The probability of blanking error as well as the bit error rate expressions with and without the blanking device under power line communication channels are derived. Computer simulations conform to the derived analytical results proving that the proposed system outperforms the conventional DCSK when a blanking device is used. Moreover, DCSK system is compared to DPSK in presence of phase noise to show its resistance and superiority. Our findings proves that the DCSK system can be considered as a potential modulation scheme for power line communication systems. Future work will focus on optimizing the blanking threshold and analyzing the performance limits of the system.

\section{REFERENCES}

[1] A. Majumder, "Power line communication," IEEE Pot., vol. 23, no. 4, pp. 4-8, Oct 2004

[2] A. Sarafi, G. Tsiropoulos, and P. Cottis, "Hybrid wireless-broadband over power lines: A promising broadband solution in rural areas," IEEE Commun. Mag., vol. 47, no. 11, pp. 140-147, Nov. 2009.

[3] IEEE Std. 1901-2010. (2010) IEEE P 1901 Working Group, IEEE Standard for Broadband over Power Line Networks: Medium Access Control and Physical Layer Specifications. [Online]. Available: http://grouper.iee.org/groups/1901/index.html

[4] Y. Ma, P. So, and E. Gunawan, "Comparison of CDMA and OFDM systems for broadband power line communications," IEEE Trans. on Power Del., vol. 23, no. 4, pp. 1876-1885, Oct. 2008.

[5] M. Zimmermann and K. Dostert, "Analysis and modeling of impulsive noise in broad-band powerline communications," IEEE Trans. on Electromagn. Compat., vol. 44, no. 1, pp. 249-258, 2002.

[6] E. Alsusa and K. Rabie, "Dynamic peak-based threshold estimation method for mitigating impulsive noise in power-line communication systems," IEEE Trans. on Power Del., vol. 28, no. 4, pp. 2201-2208, 2013.

[7] P. Chen, L. Wang, and F. Lau, "One Analog STBC-DCSK Transmission Scheme Not Requiring Channel State Information,” Trans. Circuits Syst. I, Reg. Papers, vol. 60, no. 4, pp. 1027-1037, 2013.

[8] G. Kaddoum, F. Richardson, and F. Gagnon, "Design and analysis of a multi-carrier differential chaos shift keying communication system," IEEE Trans. on Commun., vol. 61, no. 8, pp. 3281-3291, 2013.

[9] F. Lau and C. Tse, Chaos-Based Digital Communication Systems. Springer-Verlag, 2003.

[10] G. Kaddoum, P. Chargé, and D. Roviras, "A Generalized Methodology for Bit-Error-Rate Prediction in Correlation-Based Communication Schemes Using Chaos," IEEE Commun. Letters, vol. 13, no. 8, pp. 567 $-569,2009$.

[11] Y. Xia, C. Tse, and F. Lau, "Performance of differential chaos-shiftkeying digital communication systems over a multipath fading channel with delay spread," Trans. Circuits Syst. II, Exp. Briefs, vol. 51, no. 12, pp. 680-684, Nov. 2004.

[12] M. Ghosh, "Analysis of the effect of impulse noise on multicarrier and single carrier QAM systems," IEEE Trans. on Commun., vol. 44, no. 2, pp. 145-147, Feb 1996. 